\title{
Paleoicnologia do Siluro-Devoniano do estado do Paraná e a obra de John Mason Clarke
}

\author{
Paleoicnhology of the Silurian-Devonian from Paraná State \\ (southern Brazil) and the work of John Mason Clarke
}

Paleoicnología del Siluro-Devónico del estado de Paraná y la
obra de John Mason Clarke

\author{
Daniel Sedorko \\ dsedorko@gmail.com \\ Universidade Estadual de Ponta Grossa \\ Renata Guimarães Netto \\ nettorg@unisinos.br \\ Universidade do Vale do Rio dos Sinos \\ Elvio Pinto Bosetti \\ elvio.bosetti@pq.cnpq.br \\ Universidade Estadual de Ponta Grossa
}

Resumo: As camadas do Siluro-Devoniano paranaense são ricas em icnofósseis e muito se estudou sobre o tema desde a obra pioneira de John Mason Clarke, em 1913. Este trabalho apresenta um histórico dos estudos paleoicnológicos desenvolvidos em depósitos siluro-devonianos do estado do Paraná desde a obra de Clarke. A maior parte dos trabalhos enfocou depósitos da Formação Furnas e deu ênfase às características icnotaxonômicas, embora aspectos icnofaciológicos tenham sido considerados. Mesmo que discreta, a contribuição de Clarke para a icnologia do Siluro-Devoniano paranaense foi relevante por abordar um tema que ainda não possuía destaque nas geociências no início do século XX.

Palavras-chave: Icnofósseis. Siluriano. Devoniano. Bacia do Paraná.

Abstract: The Silurian-Devonian beds of Paraná State (southern Brazil) are notoriously rich in ichnofossils and several ichnological studies were carried on since the work of John Mason Clarke, in 1913. This study presents the historical record of the ichnological research in the Silurian-Devonian beds of the Paraná State. Most of the studies were focused on the Furnas Formation and emphasized preferentially the ichnotaxonomic characteristics, although some ichnofaciological aspects have been considered. Even being discrete, the contribution of Clarke to the ichnology of Silurian-Devonian beds from Paraná State was relevant to address an issue that was not yet featured in the earth sciences in the early $20^{\text {th }}$ century.

Keywords: Trace fossils. Silurian. Devonian. Paraná Basin. 
Resumen: Las capas del Siluro-Devónico del estado de Paraná (sur de Brasil) presentan abundancia de trazas fósiles, y mucho se conoce sobre el tema desde la contribución pionera realizada por John Mason Clarke, en 1913. Este trabajo presenta una historia de los estudios paleoicnológicos desarrollados en depósitos del Siluro-Devónico de Paraná desde la obra de Clarke. La mayor parte de los estudios se ha centrado en los depósitos de la Formación Furnas, destacando mayormente las características icnotaxonómicas de las trazas fósiles, aunque se han considerado aspectos icnofaciológicos. Aunque modesta, la contribución de Clarke para icnología del Siluro-Devónico del estado de Paraná ha sido relevante por abordar un tema que todavía no era destacado en las ciencias de la tierra a principios del siglo XX.

Palabras claves: Trazas fósiles. Silúrico. Devónico. Cuenca del Paraná.

\section{INTRODUÇÃO}

A icnologia estuda as interações entre os organismos e o substrato, com ênfase no modo em que os animais e as plantas deixam o registro de tal atividade (EKDALE et al., 1984). Essas interações são estudadas pela Neoicnologia em ambientes modernos e pela Paleoicnologia no registro fóssil. Seilacher (1964) sintetizou que icnofósseis são estruturas sedimentares resultantes da atividade biológica, e nesse sentido, Bromley (1996) apontou que os icnofósseis fornecem uma riqueza de informações sobre a ação da fauna endobentônica, correspondendo ao registro do comportamento desses animais. Sendo assim, considerando o caráter singenético e a geração de uma fábrica bastante específica, os icnofósseis são ferramentas úteis para se compreender a paleoecologia dos processos paleodeposicionais (NETTO, 2001). Embora apresentem pouco valor bioestratigráfico devido ao longo range temporal e faciológico, são aplicáveis em correlações de superfícies isócronas de uma mesma bacia, correlações paleogeográficas entre bacias contemporâneas, ou ainda para comparações paleoecológicas de sucessões sedimentares de idades diferentes (NETTO, 2001; BUATOIS et al., 2002). O zoneamento paleoambiental a partir da Icnologia permite, principalmente, inferir flutuações no nível de base, reconhecer aspectos da gênese dos depósitos sedimentares relacionados a parâmetros paleoecológicos e auxiliar na montagem do arcabouço de sequências.

As camadas do Siluro-Devoniano paranaense são reconhecidamente ricas em icnofósseis e muito se produziu sobre o tema desde a obra pioneira de John Mason Clarke, de 1913, intitulada "Fósseis devonianos do Paraná". Nessa perspectiva, este trabalho realiza um histórico dessa produção para identificar a principal abordagem das pesquisas paleoicnológicas nas unidades em apreço.

\section{CONTEXTO GEOLÓGICO}

A Bacia do Paraná ocorre em uma vasta região no centro-leste da América do Sul. No Brasil, essa bacia é composta por dois depocentros sedimentares, i.e. Sub-bacia de 
Alto Garças, ao Norte, e Sub-bacia de Apucarana, ao Sul. Possui uma área de aproximadamente $1.600 .000 \mathrm{~km}^{2}$, distribuídos no centro-sul do Brasil, Paraguai, Uruguai e nordeste da Argentina (MILANI et al., 2007).

Segundo Milani \& Ramos (1998), a Bacia do Paraná originou-se como um golfo aberto para o Panthalassa e, com o tempo, tornou-se uma depressão intracratônica aprisionada no interior do Gondwana. Os estratos do Siluro-Devoniano são reconhecidos no registro estratigráfico da Bacia do Paraná como uma unidade de ampla escala (Supersequência Paraná; MILANI et al., 2007) e se caracterizam por condições marinhas representadas por sucessões sedimentares que definem ciclos transgressivos-regressivos ligados a oscilações do nível relativo do mar. As unidades litológicas do Devoniano paranaense consistem em depósitos sedimentares siliciclásticos em ambiente de mar epicontinental, localizado entre $70^{\circ}$ e $80^{\circ}$ de paleolatitude Sul à época da deposição, com clima temperado a subpolar (COOPER, 1977; SCOTESE \& MCKERROW, 1990; ISAACSON \& SABLOCK, 1990). São interpretadas como dominantemente episódicas, com a preservação dos eventos deposicionais de maior magnitude, caracterizados por depósitos de águas rasas de fácies praial e de águas mais profundas de costa afora.

Lange \& Petri (1967) propuseram a divisão litoestrafigráfica do Siluro-Devoniano da Bacia do Paraná nas formações Furnas e Ponta Grossa, esta subdividida em Membros Jaguariaíva, Tibagi e São Domingos. Resgatando o trabalho de Oliveira (1912), Grahn et al. (2013) redefiniram essas unidades, denominando-as, da base para o topo, de Formações Furnas, Ponta Grossa e São Domingos.

\section{HISTÓRICO DOS ESTUDOS PALEOICNOLÓGICOS}

Os primeiros registros de icnofósseis no Siluro-Devoniano do Paraná foram realizados por Oliveira $(1912$; 1927) para a Formação Furnas, identificados como Arenicolites isp., e por Clarke (1913), que registrou a ocorrência de tubos de vermes nas camadas superiores da formação. Carvalho (1941) também se referiu à presença de Arenicolites na unidade em questão. Essa ocorrência foi revisada por Ciguel (1996), que atribuiu o espécime ilustrado por Carvalho (1941) ao material utilizado como parátipo de Fraena furnai por Lange (1942). A icnoespécie Fraena furnai foi atribuída, segundo Ciguel (1996), pelas características morfológicas do icnofóssil serem semelhantes às descritas para Fraena ROUALT, 1850, sendo a designação da icnoespécie em alusão a sua ocorrência na Formação Furnas. Lange (1942) ainda relatou a ocorrência de bioturbações referidas como Bilobites e interpretadas como resultado da atividade de anelídeos, crustáceos ou equinodermos. Contudo, Orbigny (1953), posteriormente, incluiu Bilobites no icnogênero Cruziana. Petri (1948) também mencionou a ocorrência de bioturbações na Formação Furnas.

Lange (1954) novamente mencionou a ocorrência de Fraena furnai na mesma formação. Em estudo posterior, Lange \& Petri (1967) alteraram Fraena furnai para Rouaultia furnai, ainda que sem explicitar as causas dessa transferência. Fernandes \& Netto (1985) identificaram as ocorrências como pertencentes ao icnogênero Didymaulichnus. Depois, 
Ciguel \& Aceñolaza (1986), ao abordar a icnologia da Formação Furnas, esclareceram a problemática da nomenclatura citando Häntszchel (1975), que recomendou a denominação Didymaulichnus em lugar de Rouaultia, já que esse nome foi atribuído previamente a um gênero de molusco. Ciguel \& Aceñolaza (1986) ainda identificaram os icnogêneros Lockeia, Skolithos, Rusophycus e Cruziana, e as icnoespécies Palaeophycus tubularis e Palaeophycus alternatus na Formação Furnas, além de atribuir a ocorrência relatada por Ciguel \& Godoy (1985) para a localidade de Cerro do Purunã como pertencente ao icnogênero Palaeophycus.

Aceñolaza \& Ciguel (1987) compararam os icnofósseis das formações Balcarce (Argentina) e Furnas e sugeriram o posicionamento de ambas no período Siluriano, devido à associação icnofossilífera encontrada. Ainda que icnofósseis não sejam bons datadores cronoestratigráficos, são excelentes bioindicadores paleoambientais e permitiram, aos autores, inferir um paleoambiente marinho para as camadas estudadas em condições mais profundas que aquelas expressas pela Icnofácies Skolithos.

Os autores ainda sugeriram que Didymaulichnus furnai correspondesse, de fato, a Didymaulichnus lyelli. Ciguel \& Aceñolaza (1991) novamente atribuíram as ocorrências de Fraena furnai e Rouaultia furnai ao icnogênero Didymaulichnus, mas Borghi \& Schubert (1992) propuseram o icnogênero Furnasichnus para substituir essa denominação. Por fim, Ciguel (1996) estabeleceu Didymaulichnus lyelli para as ocorrências anteriormente referidas e propôs, assim, que os espécimes anteriormente descritos como F. furnai, R. furnai e a D. furnai também fossem sinonimizados a D. lyelli.

Rodrigues et al. $(1988 ; 1989)$ citaram a ocorrência de icnofósseis para a Formação Furnas na região do Alagados (Ponta Grossa), mas não identificaram ou descreveram o material. Ciguel (1996), ao referir-se a essa ocorrência, sugeriu que a localidade visitada pelos autores supracitados correspondesse às áreas percorridas por Oliveira (1912; 1927) e Lange (1942), sendo, portanto, conhecidas desde 1912 como "Usina Velha". Ciguel \& Aceñolaza (1988) apontaram, ainda, a ocorrência de Conosthicus para essa localidade.

Associando as ocorrências icnofossilíferas com dados sedimentológicos, Bergamaschi (1992) interpretou os depósitos da Formação Furnas como de shoreface superior. $\mathrm{O}$ autor reconheceu diversos icnogêneros, tais como Rusophycus, Palaeophycus, Planolites, Didymaulichnus e Monocraterion. Essa associação foi interpretada como Icnofácies Skolithos, corroborando a interpretação paleoambiental proposta para o topo da unidade. É mister observar, contudo, que o padrão da associação icnofossilífera descrita por Bergamaschi (1992) - dominância de escavações de alimentação de detritívoros e filtradores, presença de pistas relacionadas ao deslocamento de moluscos e de escavações rasas de repouso de trilobitas - representa, de fato, uma associação de Icnofácies Cruziana proximal (e.g., BROMLEY, 1996; PEMBERTON et al., 2001; BUATOIS \& MÁNGANO, 2011).

Ao analisar testemunhos de sondagem dessa formação, Borghi $(1993 ; 1994)$ reconheceu a Icnofácies Skolithos, apontada pela presença de Skolithos, Cylindrichnus e Lockeia, e a Icnofácies Cruziana, inferida pela associação de Palaeophycus, Rusophycus, Scolicia e Furnasichnus. O autor utilizou esses dados para corroborar uma interpretação de paleoambiente deposicional de marinho raso. Posteriormente, Dino \& Rodrigues (1995) 
citaram a ocorrência de Monocraterion e Planolites, e, embasados nessas ocorrências, Mussa et al. (1996) também se referiram à Icnofácies Skolithos. Cabe ressaltar que a atribuição a Scolicia feita por Borghi $(1993 ; 1994)$ deve ser revista, já que esse icnogênero não possui expressão paleozoica, ocorrendo apenas a partir do Mesozoico (UCHMAN, 1995; 1998).

Assine \& Góis (1996) e Assine (1996; 1999) reconheceram os icnogêneros Planolites, Palaeophycus, Rusophycus, Cruziana, Arenicolites e Didymaulichnus, relacionando-os à Icnofácies Cruziana. Nesse contexto, Assine (1996; 1999) utilizou os icnogêneros Rusophycus e Cruziana (figura 1) para corroborar o caráter marinho de alguns intervalos da Formação Furnas, uma vez que são interpretados, respectivamente, como marca de repouso e deslocamento de trilobitas (organismos marinhos indicadores de salinidade normal) para o baixo Paleozoico.

Figura 1: Icnofósseis da Formação Furnas

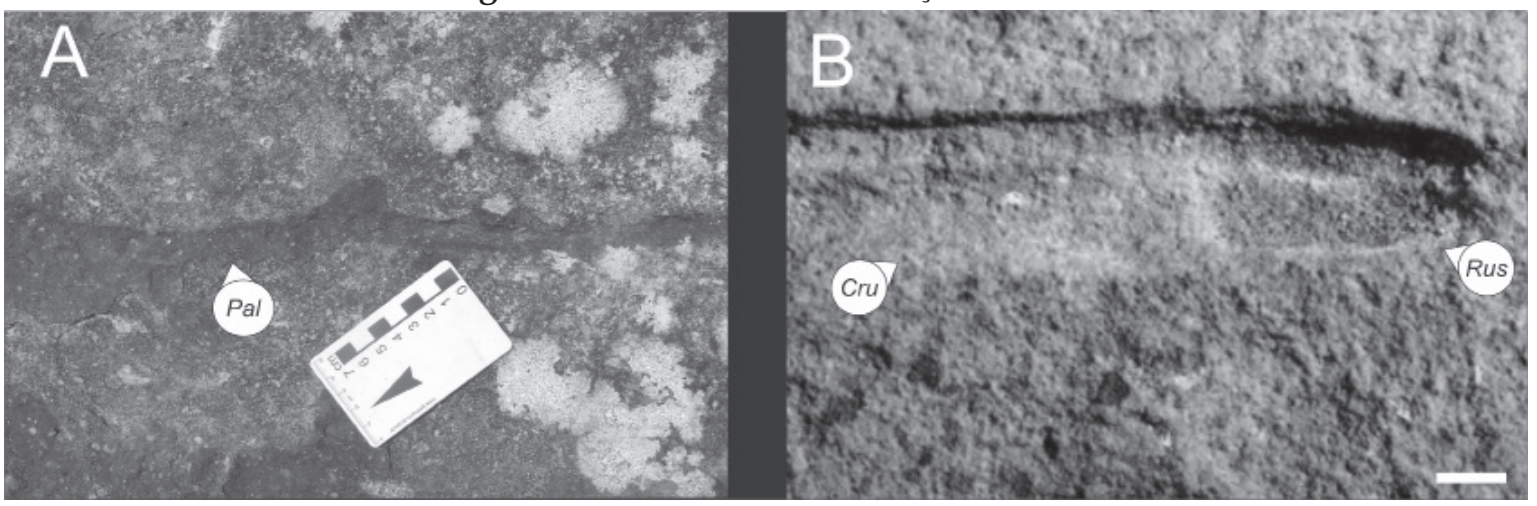

Legenda: A - Palaeophycus isp. B - Cruziana acacensis e Rusophycus isp. (escala $1 \mathrm{~cm}$ ) Modificado de Assine (1999)

Ciguel et al. (1996) identificaram Planolites, Palaeophycus, Didymaulichnus, Arenicolites e Skolithos na localidade de Sítio Cercado, Palmeira, PR. Os autores apontaram que a icnofauna encontrada indica características paleobatimétricas correspondentes à Icnofácies Skolithos. Contudo, ao considerar as ocorrências de Didymaulichnus e de traços indicativos de Rusophycus, aventaram, com ressalvas, uma posição de transição com a Icnofácies Cruziana. Associando dados sedimentológicos, os autores sugeriram tratar-se de deposição em contexto plataformal, em zona intermaré sujeita a tempestades ocasionais, com esporádica ação fluvial. Nessa mesma localidade, Moreira et al. (1998) relataram a presença de Arthrophycus.

Fernandes (1996), ao abordar o baixo Paleozoico da Bacia do Paraná, citou, para as formações Furnas e Ponta Grossa (sensu LANGE \& PETRI, 1967), a presença dos icnogêneros Bifungites, Cylindrichnus, Didymaulichnus, Furnasichnus, Lockeia, Monocraterion, Notopus (cf. Asteriacites), Palaeophycus, Palaeosabella, Planolites, Rusophycus, Skolithos e Zoophycos. Embora o autor não tenha registrado o icnogênero Cruziana, Ciguel \& Aceñolaza (1986) e Borghi (1993) já assinalavam sua presença para as camadas em apreço. Nesse contexto, Ciguel \& Aceñolaza (1986) discutiram a equivalência de Rusophycus imbricata e Cruziana rugosa, arguindo que Seilacher (1970) considerara Cruziana como Rusophycus. O icnogênero Conosthicus também havia sido referido por Ciguel \& Aceñolaza (1988; 1989) 
e Ciguel \& Netto (1989), mas não foi mencionado por Fernandes (1996), que referiu-se à ocorrência como Cylindrichnus concentricus.

Resgatando a discussão relacionada à nomenclatura, Borghi \& Fernandes (2001) propuseram formalmente o icnofóssil Furnasichnus langei, que até o presente é restrito à Formação Furnas. Abelha et al. (2005) sugeriram um contexto deposicional de "lagunas" costeiras ou baixios entre sandwaves para as ocorrências de Rusophycus da Formação Furnas nas regiões de Ponta Grossa, Tibagi e Ventania, cujos produtores (trilobitas) teriam sido levados por eventos de tempestade. Os autores consideram que teria decorrido tempo suficiente para que se produzissem os icnofósseis de repouso (Cubichnia) em questão. Desse modo, por representarem exceções ao paleoambiente, não se poderia atribuir tais achados propriamente à Icnofácies Cruziana, como sugerido por Ciguel et al. (1996).

Assine (1999), Tognoli et al. (2002; 2003), Seilacher (2007) e Tognoli \& Netto (2010) reconheceram Cruziana acacensis, Rusophycus acacensis, ?Psammichnites e Thalassinoides para a Formação Furnas. Netto et al. (2012) sugeriram trilobitas, crustáceos e moluscos como os potenciais produtores desses icnofósseis e alocaram essa suíte de traços fósseis em um contexto de Icnofácies Cruziana proximal. Para as camadas de transição, os autores ainda assinalaram a ocorrência de duas suítes distintas, em que a principal é composta por Rosselia, Cylindrichnus e Lockeia e subordinadamente Skolithos, Arenicolites, Thalassinoides, Palaeophycus, Planolites e Teichichnus. A segunda suíte é dominada por Rosselia socialis. Para os autores, as duas suítes sugerem um contexto de Icnofácies Cruziana em contexto de shoreface inferior.

Se comparados com a Formação Furnas, os trabalhos de escopo icnológico nas formações Ponta Grossa (sensu LANGE \& PETRI, 1967) ou Ponta Grossa e São Domingos (sensu GRAHN et al., 2013) são relativamente mais recentes. Clarke (1913) abordou as bioerosões em moluscos bivalves, mas outros estudos surgiram apenas a partir da década de 1980.

Leonardi $(1982 ; 1983)$ relatou a ocorrência de uma provável pegada de anfíbio Ichthyostegalia. Essa ocorrência foi denominada Notopus petri e sua interpretação pressupôs uma ampla distribuição para a origem dos anfíbios durante o Devoniano. Porém Roček \& Rage (1994), ao revisarem o material e considerarem o contexto marinho para a Formação Ponta Grossa, assumiram tratar-se de um exemplar incompleto de Asteriacites, produzido por equinodermas asteroides. Fernandes (1996) adotou essa interpretação, invalidando Notopus petri.

Campanha (1985) analisou a ocorrência de Zoophycos, abundante na Formação Ponta Grossa, e utilizou esse icnotáxon como indicador de paleoambiente de offshore externo, ainda que com ressalvas. No mesmo ano, Fernandes \& Melo (1985) descreveram a icnoespécie Bifungites paranaensis e identificaram a ocorrência de Bifungites cruciformes nos depósitos dessa unidade. Posteriormente, Castro (1988) mencionou e ilustrou Zoophycos para as camadas em questão.

Abelha et al. (2007) identificaram, nessas camadas, as Icnofácies Zoophycos, Cruziana arquetípica, Cruziana proximal, Cruziana empobrecida e Glossifungites. Na Icnofácies Zoophycos, os autores identificaram os icnogêneros Helminthopsis e Zoophycos. 
A Icnofácies Cruziana arquetípica é localmente composta por Asterosoma, Palaeophycus, Planolites, Rhizocorallium e Teichichnus, representando um contexto paleodeposicional de mar raso de costa afora, abaixo do nível de ação de ondas de tempo bom, porém dentro do nível de base de ação de ondas de tempestade. A Icnofácies Cruziana proximal, segundo os autores, é assinalada pela presença de Skolithos, Lockeia e Arenicolites, indicando um aumento da energia do meio e caracterizando um paleoambiente mais proximal. A Icnofácies Cruziana empobrecida é composta por Schaubcylindrichnus, Asterosoma, Planolites, Teichichnus, Zoophycos e estruturas de escape, que caracterizam paleoambiente de mar raso de costa afora sujeito a ações de tempestade com influência deltaica. Por fim, os autores reconheceram a Icnofácies Glossifungites, na qual ocorre Thalassinoides, identificando, a partir dela, um provável evento de regressão forçada dentro do contexto transgressivo geral dessa sequência deposicional.

Perassa \& Silva (2009) e Perassa et al. (2009), ao analisarem ocorrências da Formação Ponta Grossa (intervalo neo-Praguiano/eo-Emsiano) na região de Jaguariaíva, cometeram o equívoco de identificar fósseis corporais de Australospirifer iheringi como icnofósseis.

Bosetti \& Silva (2009) e Bosetti et al. (2010) registraram o icnogênero Phycosiphon. A associação de grandes quantidades de Phycosiphon (figura 2.B) com fósseis corporais representantes da fauna relictual de tamanho subnormal (Efeito Lilliput de BOSETTI et al., 2011) corroborou a hipótese de que o declínio da produção primária, causada pela transgressão Eifeliana/Givetiana, propiciou espaços ecológicos vazios logo ocupados por imigrantes que, mesmo sob condições desfavoráveis, teriam suas necessidades alimentares ainda atendidas. Nesse contexto, os autores apontaram que o produtor poderia ser considerado um imigrante bem adaptado às novas condições de estresse ecológico.

Netto et al. (2012) sintetizaram as principais ocorrências icnofossilíferas para o Fanerozoico da Bacia do Paraná e, além da revisão dos trabalhos que abordaram o tema, ainda identificaram seis suítes principais para o Devoniano paranaense (Givetiano), relatando a ocorrência de Asterosoma, Chondrites, Cylindrichnus, Diplocraterion, Gordia, Halopoa, Helminthopsis, Hemidallia, ?Lennea, Lingulichnus, Lockeia, Macaronichnus (figura 2.A), Palaeophycus, Rhizocorallium, Rosselia, Skolithos, Taenidium satanassi e Zoophycos, que representam a Icnofácies Cruziana (figura 2.C). 
Figura 2: Icnofósseis do intervalo Neo-Emsiano/Eo-Givetiano

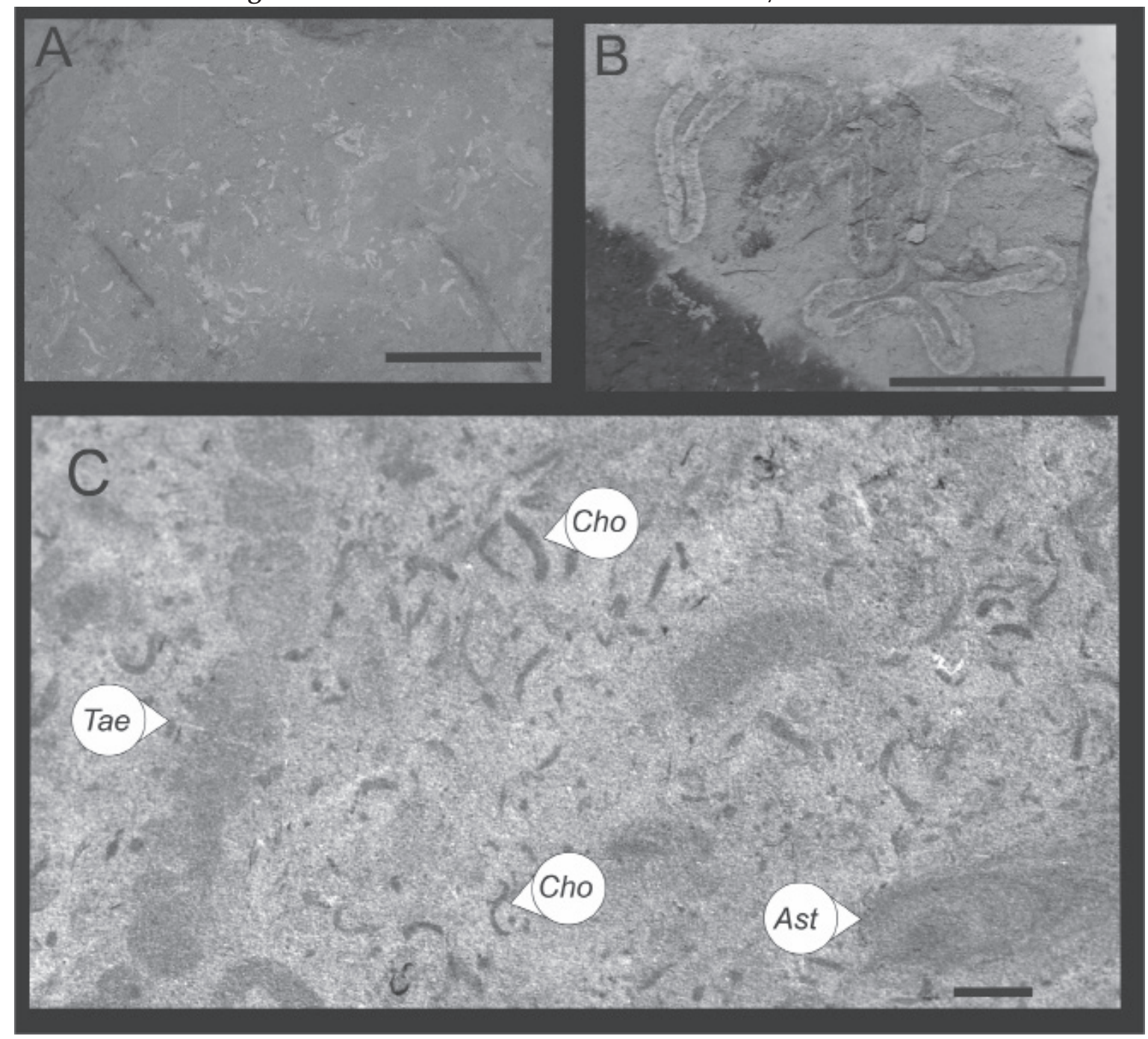

Legenda: A - suíte de Macaronichnus (escala $10 \mathrm{~cm}$ ). B - Phycosiphon isp. (escala $1 \mathrm{~cm})$. C - Taenidium satanassi, Chondrites e Asterosoma (escala $1 \mathrm{~cm}$ )

\section{ICNOLOGIA NA OBRA DE JOHN MASON CLARKE}

Clarke (1913), referindo-se ao "Arenito Furnas", citou a ausência de fósseis ou traços fósseis, salvo tubos de vermes nas camadas superiores. Para a Formação Ponta Grossa, o autor identificou algumas amostras contendo tubos quitinosos alongados e encurvados, ligeiramente cônicos e achatados denominados Serpulites, então atribuídos à atividade de anelídeos (figura 3). Uma vez que Serpulites sica já havia sido descrito para as camadas de Bokkeveld (África do Sul), ainda que de tamanho menor, o autor não viu problemas em atribuir as ocorrências de Ponta Grossa a essa denominação.

Nessa obra, registrou-se também a ocorrência de perfurações em conchas dos moluscos Palaeoneilo magnifica, Leptodomus capricornus e Ptomatis moreirai atribuídas à atividade de uma esponja do gênero Clionolithus, cujos tubos se apresentam em grupos paralelos com aspecto reto claviforme sendo denominadas Clionolithus priscus (figura 3). O 
autor assinalou que essas perfurações são frequentes em outras conchas de fauna similar, mas ainda não haviam sido registradas em faunas austrais. McCoy (1855 apud CAMERON, 1969) havia assinalado a presença de pequenas perfurações em conchas de Pterinea demissa, denominando de Vioa prisca e atribuindo a ocorrência à atividade de perfuração de esponjas. Posteriormente, Clarke (1921) considerou que Clionolithus priscus teria sido resultado da ação de anelídeos perfuradores de concha, propondo, assim, a denominação Palaeosabella prisca, que incluiria ainda a ação de perfuração por esponjas e organismos vermiformes. Fenton \& Fenton (1932) reafirmaram Vioa prisca como resultado da ação de esponjas e, devido a semelhanças e critérios de prioridade de nomes, transferiram para Topsentia CLARKE 1921, denominando como Topsentia (?) prisca. Considerando essa ambiguidade, Cameron (1969) propôs, então, a denominação Vermiforichnus clarkei, substituindo as anteriores.

Figura 3: Icnofósseis representados por Clarke (1913)

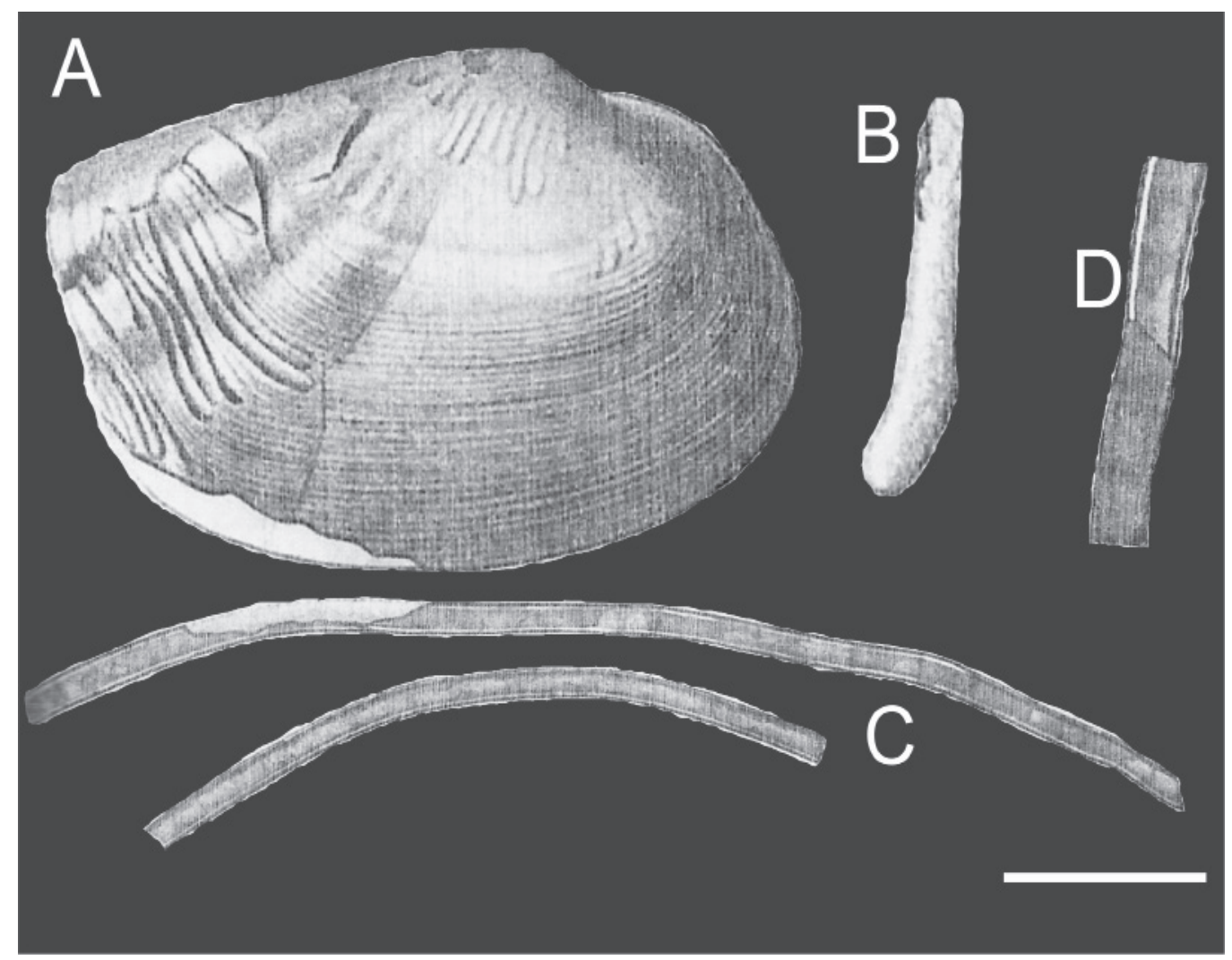

Legenda: A - Palaeoneilo magnifica crivada com Clionolithus priscus (= Palaeosabella prisca). B - Detalhe de Palaeosabella prisca (aumentado 2x). C - Tubos quitinosos, achatados, com bordas engrossadas correspondendo a Serpulites sica. D - Detalhe de Serpulites sica (aumentado 2x). Adaptado de Clarke (1913). Escala: A e C - 1 cm; B e D - 0,5 cm 
Pemberton et al. (1980) descreveram Palaeosabella para o Devoniano referindo-se como Trypanites. Esse icnogênero já havia sido descrito por Mägdefrau (1932), levantando dúvidas, então, quanto à atribuição do material de origem devoniana. Ao examinar o material tipo, Plewes (1996) concluiu que a denominação Palaeosabella CLARKE 1921 é válida, caracterizando, assim, as perfurações longas e tubulares, e Bromley (2004) ressaltou que, embora Palaeosabella e Trypanites sejam semelhantes, o segundo representa perfuração cilíndrica, enquanto que Palaeosabella expande-se distalmente como um cone agudo. Deste modo, a diagnose deve ser criteriosa para se evitar futuras confusões relacionadas à nomenclatura.

Palmer et al. (1997) sugeriram que Trypanites seria dominante em depósitos pós-Paleozoico, enquanto que Palaeosabella estaria mais restrito ao Paleozoico. Os estudos de Bromley \& D'Alessandro (1987) e de Cole \& Palmer (1999) corroboraram essa interpretação, assinalando que Trypanites é comum apenas a partir do Mesozoico.

Nesse contexto, Palaeosabella prisca é o único icnogênero associado à bioerosão no Devoniano do estado do Paraná. Zabini et al. (2006a; 2006b) relataram a ocorrência de perfurações circulares em valvas de lingulídeos, mas sem apresentarem identificação icnotaxonômica. O registro de bioerosões é raro para os macroinvertebrados marinhos do Devoniano paranaense.

\section{CONSIDERAÇÕES FINAIS}

Ainda que Oliveira (1912) tenha se referido à presença de Arenicolites para a Formação Furnas, é na obra de Clarke (1913) que aparece a primeira discussão relacionada a estruturas de bioerosão para o estado do Paraná. Como anteriormente exposto, o autor também assinala a presença de tubos de verme e Serpulites sica para o Siluro-Devoniano paranaense. A obra de John Mason Clarke figura como marco no conhecimento paleontológico do Paraná.

Neste século de pesquisa, muitos autores contribuíram para o conhecimento icnológico do estado, mas a maioria dos trabalhos concentrou-se na Formação Furnas. Embora alguns estudos tenham se utilizado de interpretações icnofaciológicas para essa formação, a maior ênfase dos trabalhos abordou características icnotaxonômicas. A abundância de trabalhos para $o$ intervalo Pridoliano/Praguiano, se confrontada com as unidades superiores pertencentes ao intervalo Neo-Praguiano/Eo-Givetiano, pode estar relacionada à ausência de fósseis corporais preservados no primeiro. Por outro lado, a produção científica relacionada aos icnofósseis do segundo intervalo é extremamente baixa se comparada aos estudos desenvolvidos com os macrofósseis de invertebrados dessas mesmas unidades. Embora aspectos icnofaciológicos tenham sido considerados, a tendência dos trabalhos foi generalista, carecendo de estudos icnológicos detalhados para essas seções.

Em síntese, ainda que muito se tenha produzido para o Siluro-Devoniano do Paraná, pouco se conhece da icnologia relacionada aos processos paleodeposicionais e aos paleoambientes refletidos pelas unidades deposicionais da Supersequência Paraná, sendo necessários novos estudos, principalmente para as camadas superiores. 


\section{AGRADECIMENTOS:}

Os autores agradecem ao Conselho Nacional de Desenvolvimento Científico e Tecnológico - CNPq (305208/2010-1; 150239/2011-4; 401796/2010-8) e à Coordenação de Aperfeiçoamento de Pessoal de Nível Superior (CAPES).

\section{REFERÊNCIAS:}

ABELHA, Mariana; BORGHI, Leonardo; FERNANDES, Antônio Carlos Sequeira. Rusophycus do Devoniano da bacia do Paraná. In: CONGRESSO LATINO-AMERICANO DE PALEONTOLOGIA, 6, Aracajú. Anais do VI Congresso Latino-Americano de Paleontologia, Aracajú, 2005.

ABELHA, Mariana; BORGHI, Leonardo; FERNANDES, Antônio Carlos Sequeira. Análise Icnológica da Formação Ponta Grossa (Devoniano) em Afloramentos da Borda Leste da Bacia do Paraná. In: JORNADA GIULIO MASSARANI DE INICIAÇÃO CIENTÍFICA, ARTÍSTICA E CULTURAL DA UFRJ, 28, Rio de Janeiro. Livro de Resumos. Rio de Janeiro: Anuário do Instituto de Geociências - UFRJ, 2007. p. 234.

ACEÑOLAZA, Florencio Gilberto; CIGUEL, José Henrique Godoy. Analisis comparativo entre las formaciones Balcarce (Argentina) y Furnas (Brasil). In: CONGRESSO GEOLÓGICO ARGENTINO, 10, San Miguel de Tucamán. Actas, San Miguel de Tucamán: SAG, t1, 1987. p. 299-305.

ASSINE, Mário Luis. Aspectos da estratigrafia das sequências pré-carboníferas da Bacia do Paraná no Brasil. 1996. Tese (Doutorado em Geologia Sedimentar), USP, São Paulo, SP. 207 p.

ASSINE, Mário Luis. Fácies, icnofósseis, paleocorrentes e sistemas deposicionais da Formação Furnas no flanco sudeste da Bacia do Paraná. Revista Brasileira de Geociências, v. 29, p. 357-370, 1999.

ASSINE, Mário Luis; GÓIS, José Roberto de. Traços fósseis de trilobita na Formação Furnas, Bacia do Paraná, Brasil. In: SIMPÓSIO SULAMERICANO DO SILURO-DEVONIANO, 1, Ponta Grossa. Anais. Ponta Grossa: UEPG-UFPR, v. 1, 1996. p. 371-373.

BERGAMASCHI, Sérgio. Análise sedimentológica da Formação Furnas na faixa de afloramentos do flanco norte do arco estrutural de Ponta Grossa, Bacia do Paraná, Brasil. 1992. Dissertação (Mestrado em Geologia) Instituto de Geociências, UFRJ, Rio de Janeiro, RJ. 172 p.

BORGHI, Leonardo. Caracterização e análise faciológicas da Formação Furnas (Prídoli-Devoniano inferior) em afloramentos do bordo leste da bacia sedimentar do Paraná, Estado do Paraná, Brasil. 1993. Dissertação (Mestrado em Geologia) Instituto de Geociências, UFRJ, Rio de Janeiro, RJ. 227 p.

BORGHI, Leonardo. Icnofácies da Formação Furnas no estado do Paraná. Anais da Academia Brasileira de Ciências, v. 66, n. 1, p. 121, 1994.

BORGHI, Leonardo; SCHUBERT, Gilberto. Furnasichnus langei, ichnog. et ichnosp. nov., and its relation to other trace fossils from the Devonian of Paraná State, Brazil. Anais da Academia Brasileira de Ciências, v. 64, p. 418, 1992.

BORGHI, Leonardo; FERNANDES, Antônio Carlos Sequeira. A new trace fossil from the Devonian of the Parana State (Parana Basin), Brazil. Boletim do Museu Nacional, Geol. v. 58, p. 1-12, 2001.

BOSETTI, Elvio Pinto; SILVA, Rafael Costa da. Primeira ocorrência de Phycosiphon Von Fischer-Ooster, 1858 na Formação Ponta Grossa (Devoniano, Bacia do Paraná) e considerações sobre mudanças ecológicas na passagem Eifeliano-Givetiano. In: REUNIÃO ANUAL DA SOCIEDADE BRASILEIRA DE PALEONTOLOGIA - PALEO, 2009, 11, Curitiba. Boletim de Resumos, Curitiba. 2009. p. 10.

BOSETTI, Elvio Pinto; HORODYSKI, Rodrigo Scalise; COMNISKEY, Jeanninny Carla. Phycosiphon and the biotic crisis in the latest Eifelian/earliest-Givetian boundary, Paraná Basin, Brazil. In: SIMPOSIO LATINOAMERICANO DE ICNOLOGIA, 1, São Leopoldo. Resúmenes. São Leopoldo: Unisinos, 2010. p. 26.

BOSETTI, Elvio Pinto; GRAHN, Yngve; HORODYSKI, Rodrigo Scalise; MAULLER, Paula Mendlowicz.; BREUER, Pierre; ZABINI, Carolina. An Earliest Givetian "Lilliput Effect" in the Paraná Basin, and the collapse of the Malvinokaffric shelly fauna. Paläontologische Zeitschrift. v. 85, p 49-65. March 2011. 
BROMLEY, Richard Granville. Trace fossils: Biology, taphonomy and aplications. Londres: Chapman \& Hall, 1996. $361 \mathrm{p}$.

BROMLEY, Richard Granville. A stratigraphy of marine bioerosion. In: MCILROY, Duncan(ed.). The application of ichnology to palaeoenvironmental and stratigraphic analysis. London: Geological Society, 2004. p. 455-481.

BROMLEY, Richard Granville; D'ALESSANDRO, Assunta. Bioerosion of the Plio-Pleistocene transgression of southern Italy. Rivista Italiana di Paleontologia et Stratigraphie, 93, 379-442, 1987.

BUATOIS, Luis Alberto; MÁNGANO, María Gabriela; ACEÑOLAZA, Florencio Gilberto. Trazas fósiles: Señales de comportamiento em el registro estratigráfico. Chubut: Museo Paleontológico Egidio Feruglio, 2002. 382p.

BUATOIS, Luis Alberto; MÁNGANO, María Gabriela. Ichnology: Organism-Substrate Interactions in Space and Time. New York: Cambridge University Press. 2011. 358 p.

CAMERON, Barry. New name for Palaeosabella prisca (McCoy), a Devonian worm-boring, and its preserved probable borer. Journal of Paleontology, 43, p. 189-192, 1969.

CAMPANHA, Vilma Alves. O significado do icnofóssil Zoophycos na sedimentação da Formação Ponta Grossa (D) Bacia do Paraná. Anais da Academia Brasileira de Ciências, 57, 1985.

CARVALHO, Paulino Franco de. O Devoniano do Paraná. Boletim DNPM-DGM, 109, 9-27, 1941.

CASTRO, Joel Carneiro de. Sedimentology. stratigraphy and paleontology of the Gondwana sequence of the Parana Basin. In: INTERNATIONAL GONDWANA SYMPOSIUM, 17, Sao Paulo, Brazil. Guidebook. Excursion A2: Rio de Janeiro. Petroleo Brasilerio S.A., 1988. p. 100.

CIGUEL, José Henrique Godoy; ACEÑOLAZA, Florencio Gilberto. Icnologia da Formação furnas (Paleozoico Médio), Bacia do Paraná. Anais da Academia Brasileira de Ciências, 58, 4, p. 595-596, 1986.

CIGUEL, José Henrique Godoy. A presença de Didymaulichnus lyelli na Formação Furnas (Siluro-Devoniano, Flanco Oriental da Bacia do Parana) - Revisão dos icnofósseis referidos de 1912 a 1989. In: SIMPÓSIO SULAMERICANO DO SILURO-DEVONIANO, 1, Ponta Grossa. Anais. Ponta Grossa: UEPG-UFPR, v. 1, 1996.

CIGUEL, José Henrique Godoy; ACEÑOLAZA, Florencio Gilberto. Nota sobre a ocorrência de Conostichus na Formação Furnas, estado do Paraná, Brasil. In. REUNION DEL PROYETO 270 IGCP, 1, Annaes Eventos Del Paleozoico Inferior em Latinoamerica, Santiago, Chile, 1988.

CIGUEL, José Henrique Godoy; ACEÑOLAZA, Florencio Gilberto. Conosthicus na Formação Furnas (flanco oriental) no estado do Paraná. In: CONGRESSO BRASILEIRO DE PALEONTOLOGIA, 11, Curitiba. Boletim de Resumos SBP, 1989. p. 13-14.

CIGUEL, José Henrique Godoy; ACEÑOLAZA, Florencio Gilberto. Icnologia da Formação Furnas (Paleozoico Médio), Bacia do Paraná. Revista Técnica de YPFB, 12, 1, p. 145, 1991.

CIGUEL, José Henrique Godoy; GODOY, Helder de. Relatório da folha geológica de Camarinha parcial leste Cerro do Purunã. Relatório UFPR, ST-DEPARTAMENTO DE GEOLOGIA, 1985.

CIGUEL, José Henrique Godoy; NETTO, Renata Guimarães. Icnofósseis. In: ROSLER, Oscar. Paleontologia da Bacia do Paraná e Roteiro de Excursão. CONGRESSO BRASILEIRO DE PALEONTOLOGIA, 11, Curitiba. Roteiro de Campo, 1989.

CIGUEL, José Henrique Godoy; PEDREIRA, Augusto José; GOIS, José Roberto de. Os icnofósseis da localidade de Sitio Cercado, estado do Paraná, Brasil - Formação Furnas. (Siluriano-Devoniano), flanco oriental da Bacia do Paraná. In: SIMPÓSIO SULAMERICANO DO SILURO-DEVONIANO, 1, Ponta Grossa. Anais. Ponta Grossa: UEPG-UFPR, v. 1, 1996.

CLARKE, John Mason. Fósseis devonianos do Paraná. Monografia do serviço geológico e mineralógico do Brasil, 1913.

CLARKE, John Mason. Organic Dependence and Disease: Their Origin and Significance. Yale University Press, New Haven, 1921.

COLE, Alan; PALMER, Timothy. Middle Jurassic worm borings, and a new giant ichnospecies of Trypanites from the Bajocian/Dinantian unconformity, southern England. Proceedings of the Geologists' Association, 110, p. 203-209, 1999. 
COOPER, Paul. Paleolatitudes in the Devonian of Brazil and the Frasnian-Famennian mass extinction. Palaeogeography, Palaeoclimatology, Palaeoecology. 1977.

DINO Rodolfo; RODRIGUES Maria Antonieta da Conceição. Palinomorfos eodevonianos da Formação Furnas Bacia do Paraná. Anais da Academia Brasileira de Ciências, 67, p. 107-116. 1995.

EKDALE, Allan; BROMLEY, Richard Granville; PEMBERTON, Stuart George. Ichnology, Trace Fossils in Sedimentology and Stratigraphy. Society of Economic Paleontologists and Mineralogists. Short Course, 1984.

FENTON, Carroll Lane; FENTON, Mildred Adams. Boring sponges in the Devonian of Iowa. American Midland Naturalist, 13, p. 42-54, 1932.

FERNANDES, Antônio Carlos Sequeira; MELO, José Henrique Gonçalves de. Ocorrência do icnogênero Bifungites na Formação Ponta Grossa, Devoniano do estado do Paraná. Anais da Academia Brasileira de Ciências, Rio de Janeiro, 57, 2, p. 203-207, 1985.

FERNANDES, Antônio Carlos Sequeira; NETTO, Renata Guimarães. O estado atual da paleoicnologia no Paleozoico da Bacia do Paraná. In: CONGRESSO BRASILEIRO DE PALEONTOLOGIA, 9, Fortaleza. Resumo das Comunicações, Fortaleza: SBP, p. 41. 1985.

FERNANDES, Antônio Carlos Sequeira. Os icnofósseis do Ordoviciano, Siluriano e Devoniano da Bacia do Paraná. 1996. Tese (Doutorado - Programa de Pós-Graduação em Geologia), Universidade Federal do Rio de Janeiro, Rio de Janeiro, 183 p.

GRAHN, Yngve; MAULLER, Paula Mendlowicz; BERGAMASCHI, Sérgio; BOSETTI, Elvio Pinto. Palynology and sequence stratigraphy of three Devonian rock units in the Apucarana Sub-basin (Paraná Basin, south Brazil): additional data and correlation. Review of Palaeobotany and Palynology 198, p. 27-44, 2013.

HÄNTZSCHEL, Walter. Trace fossil and problematica. In: TEICHERT, Curt. (ed.) Treatise on invertebrate paleontology, Part W, Suplement 1, 1975.

ISAACSON, Peter; SABLOCK, Peter. Devonian palaeogeography and palaeobiogeography of the Central Andes. In: MCKERROW, William Stuart; SCOTESE, Christopher Robert (eds.): Palaeozoic Palaeogeography and Biogeography, Geological Society of London Memoir, 12, p. 429-433, 1990.

LANGE, Frederico Waldemar. Restos vermiformes do arenito Furnas. Arquivos do museu paranaense, 2, 1, p. 3-8, 1942.

LANGE, Frederico Waldemar. Paleontologia do Paraná. Curitiba, Comissão de comemoração do centenário do Paraná, 1954. 210 p.

LANGE, Frederico Waldemar; PETRI, Setembrino. The devonian of the Paraná Basin. Boletim Paranaense de Geociências. (21-22): p. 5-55, 1967.

LEONARDI, Giuseppe. Descoberta de pegada de um anfíbio Devoniano no Paraná. Ciencias Terra: 36-37, 1982.

LEONARDI, Giuseppe. Notopus petri, n. gen., sp.; an amphibian imprint in the Devonian of Paraná, Brazil: Geobios, v. 16, p. 233-239, 1983.

MÄGDEFRAU, Karl. liber einige Bohrgange aus dem Unteren Muschelkalk von Jena. Paläontologische Zeitschrift, 14, p.150-160, 1932.

MILANI Edison José; RAMOS Victor Alberto. Orogenias Paleozoicas no Domínio Sul-Ocidental do Gondwana e os Ciclos de Subsidência da bacia do Paraná. Revista Brasileira de Geociências, 28, 4, p. 473-484, 1998.

MILANI Edison José; MELO, José Henrique Gonçalves de; SOUZA, Paulo Alves; FERNANDES, Luiz Alberto; FRANÇA, Almério Barros. Bacia do Paraná. Boletim de Geociências da Petrobrás, Rio de Janeiro. 2007.

MOREIRA, Márcio Ivan Carvalho; BORGHI, Leonardo; FERNANDES, Antônio Carlos Sequeira. A primeira ocorrência do icnogenero Arthrophycus Hall, 1852, na Formação Furnas (Bacia do Paraná). Anais da Academia Brasileira de Ciencias, Rio de Janeiro, 70, 1, p.151, 1998.

MUSSA Diana, BORGHI Leonardo, BERGAMASCHI Sérgio, SCHUBERT Gilberto, PEREIRA Egberto, RODRIGUES Maria Antonieta da Conceição. Estudo preliminar da tafoflora da Formação Furnas, Bacia do Paraná, Brasil. Anais da Academia Brasileira de Ciências, 68. p. 65-89. 1996.

NETTO, Renata Guimarães. Icnologia e Estratigrafia de Sequências. In: RIBEIRO, Helio Jorge Portugal Severiano (ed.), Estratigrafia de Sequências - Fundamentos e Aplicações. São Leopoldo, Edunisinos, p. 219-259, 2001. 
NETTO, Renata Guimarães; TOGNOLI, Francisco Manoel Wohnrath; GANDINI, Rosana; LIMA, João Henrique Dobler; GIBERT, Jordi Maria de. Ichnology of the phanerozoic deposits of southern Brazil: sinthetic review. In: NETTO, Renata Guimarães; CARMONA, Noelia Beatriz; TOGNOLI, Francisco Manoel Wohnrath (orgs). Ichnology of Latin America: Selected Papers. Monografias da Sociedade Brasileira de Paleontologia, 2, 2012. p. 37-68.

OLIVEIRA, Eusébio Paulo de. O terreno devoniano do sul do Brasil. Annaes da Escola de Minas de Ouro Preto, 14, p. 31-41, 1912.

OLIVEIRA, Eusébio Paulo de. Geologia e recursos minerais do estado do Paraná. Monografia do serviço geológico e mineralógico, 6, 1927. $172 \mathrm{p}$.

ORBIGNY, Alcide d'. Voyage dans I'Àmérique méridionale. Partie 3 (4), 1953. 188 p.

PALMER, Timothy; PLEWES, Caryl Robin; COLE, Alan. The simple and long-ranging worm-boring Trypanites: not so simple and long-ranging after all. Geological Society of America, Abstracts with Programs, 29, 1997.269$273 \mathrm{p}$.

PEMBERTON, Stuart George, KOBLUK, David, YEO, Ross; RISK, Michael. The boring Trypanites at the SilurianDevonian disconformity in southern Ontario. Journal of Paleontology, 54, p. 1258-1266, 1980.

PEMBERTON, Stuart George; SPILA, Michelle Victoria; PULHAM, Andrew; SAUNDERS, Thomas; MACEACHERN, James; ROBBINS, Demian; SINCLAIR, Iain. Ichnology and sedimentology of shallow to marginal marine systems: Calgary, Geological Association of Canada, Short Course Notes 15, 2001. 641p.

PERASSA, Lígia Arnedo; DIAS, Angélica Corrêa; ALMEIDA, Pablo Felipe de; SILVA, José Luiz Lorenz. Icnofósseis de Jaguariaíva, PR. In: CONGRESSO DE BIÓLOGOS DO CRBIO-01, 19, São Pedro. Programa e Resumos. São Paulo: Conselho Regional de Biologia - 1ª Região (SP, MT, MS), 2009. p. 80-80.

PERASSA, Lígia Arnedo; SILVA, José Luiz Lorenz. Icnofósseis de Jaguariaíva, Formação Ponta Grossa, PR. 2009, Monografia (Licenciatura em Ciências Biológicas). Universidade Federal do Mato Grosso do Sul - Campus de Três Lagoas. 10 p.

PETRI Setembrino. Contribuição ao estudo do Devoniano paranaense. Rio de Janeiro, DNPM/DGM, 125 p. 1948.

PLEWES, Caryl Robin. Ichnotaxonomic studies of Jurassic endoliths. 1996, PhD thesis (Institute of Earth Studies), University of Wales, Aberystwyth. 313 p.

ROČEK, Zbyněk; RAGE, Jean-Claude. The presumed amphibian footprint Notopus petri from the Devonian: a probable starfish trace fossil. Lethaia, Oslo, 27, 3, p. 241-244, 1994.

RODRIGUES, Maria Antonieta da Conceição; BORGUI, Leonado; SCHUBERT, Gilberto. Novas ocorrências de icnofósseis na Formação Furnas. Anais da Academia Brasileira de Ciências, 60, 1, 109, 1988.

RODRIGUES, Maria Antonieta da Conceição; PEREIRA, Egberto; BERGAMASCHI, Sérgio. Ocorrência de Psilophytales na Formação Furnas, borda leste da Bacia do Paraná. Boletim do IG-USP, Publicação Especial, 7, p. 35-43, 1989.

SCOTESE, Christopher Robert; MCKERROW, William Stuart. Revised world maps and introduction. In: MCKERROW, William Stuart; SCOTESE, Christopher Robert (eds.) Palaeozoic, Palaeogeography and Biogeography. The Geological Society Memoir, 1990. p. 222-231.

SEILACHER, Adolf. Biogenic sedimentary structures. In: IMBRIE, John. NEWELL, Norman (eds.), Approaches to Paleoecology. Wiley and Sons, New York, p. 296-316. 1964.

SEILACHER, Adolf. Cruziana stratigraphy of non-fossiliferous Paleozoic sandstones. In: CRIMES, Thomas Peter; HARPER, John Childs (eds.), Trace Fossils. Geological Journal Special. Issue 3, p. 447-476. 1970.

SEILACHER, Adolf. Trace Fossil Analysis. Berlin, Heidelberg, New York: Springer-Verlag, 2007. 231 p.

TOGNOLI, Francisco Manoel Wohnrath; NETTO, Renata Guimarães. SLIC 2010 PreSymposium Field Trip. In: SIMPOSIO LATINOAMERICANO DE ICNOLOGÍA, 2010. Field Trip Guide, São Leopoldo, 2010.

TOGNOLI, Francisco Manoel Wohnrath; ASSINE, Mário Luis; NETTO, Renata Guimarães. Roteiro Icnológico do Grupo Paraná. Rio Claro, Universidade Estadual Paulista, 27 p, 2002. 
TOGNOLI, Francisco Manoel Wohnrath; ASSINE, Mário Luis; NETTO, Renata Guimarães. Paleodepositional interpretations based on the integration between ichnology and sedimentology, Ponta Grossa Formation, eastern Paraná Basin, Brazil. In: CONGRESSO LATINO AMERICANO DE SEDIMENTOLOGIA, 3, Belém. Actas, Belém, p. 65-67, 2003.

UCHMAN, Alfred. Taxonomy and palaeoecology of flysch trace fossils: the Marnoso-arenacea Formation and associated facies (Miocene, Northern Apennines, Italy) Beringeria, 15, 1995. p. 1-115.

UCHMAN, Alfred. Taxonomy and ethology of flysch trace fossils: a revision of the Marian Książkiewicz collection and studies of complementary material. Annales Societatis Geologorum Poloniae, 68, p. 105218, 1998.

ZABINI, Carolina; BOSETTI, Elvio Pinto; HOLZ, Michel. Presença de perfurações em lingulídeos (Brachiopoda: Inarticulata) da Sucessão Devoniana da Sub-Bacia de Apucarana, Bacia do Paraná, Brasil. In: XV ENCONTRO ANUAL DE INICIAÇÃO CIENTÍFICA E VI ENCONTRO DE PESQUISA DA UEPG, Ponta Grossa, Anais, 2006a. 4 p.

ZABINI, Carolina; BOSETTI, Elvio Pinto; HOLZ, Michel. Análise de perfurações em Lingulídeos (Brachiopoda: Linguliformea) da Sucessão Devoniana da Sub-Bacia de Apucarana, Bacia do Paraná, Brasil. In: SEMANA ACADÊMICA DOS ALUNOS DE PÓS-GRADUAÇÃO EM GEOCIÊNCIAS, 1, Porto Alegre. Anais: Porto Alegre, 2006b. 4 p.

Recebido em 23/10/2013

Aceito para publicação em 27/11/2013 